\title{
Comparison of pipette method and state of the art analytical techniques to determine granulometric properties of sediments and soils
}

\author{
FRUZSINA GRESINA ${ }^{1}$
}

\begin{abstract}
The determination of particle size distribution is a crucial issue in various fields of earth sciences (e.g., Quaternary research, sedimentology, stratigraphy, structural geology, volcanology), environmental sciences as well as diverse industrial applications (e.g., pharmaceuticals, cement industry). New measurement techniques developed as a result of industrial demands have also gained ground in environmental and Earth sciences research. The new techniques (especially laser diffraction) have enabled the particle characterisation in the broader size-range with a more detailed resolution. Still, they have to be compared with data obtained by classical methods. In light of the above, the primary aim of our research is to examine the methods of particle size determination critically. Excessive oversimplifications of particle size analyses routinely have used in paleo-environmental and paleo-climatological reconstructions, and other sedimentary studies, as well as insufficient knowledge of the background of the applied methods, distort the interpretation of the results. Over the past four decades, laser diffraction particle size analysers have proven to be practical tools of particle size characterisation. However, the shape of the natural sediment and soil particles are irregular and, therefore, affects the particle size distribution results obtained by different methods. The results of the traditional pipette method differed from laser diffraction results. The presence or absence of the pretreatments did control the differences between the two techniques. The results of Fraunhofer optical method were significantly different from Mie theory because it can detect much lower volume percentages of finer particles. Grain size results of coarse-grained samples measured by different laser diffraction devices were more comparable than the results of more clayey samples. The ratios of different sizes were changed due to the hydrochloric acid and hydrogen peroxide pretreatments. The comparison of different techniques is necessary to revaluate standards in grain size measurements which can enable the shift from conventional methods to more productive and reproducible methods. Still, light scattering techniques have not yet been able to displace classical methods in Earth sciences completely, in contrast to industrial applications.
\end{abstract}

Keywords: grain size analysis, laser diffraction, pipette method, particle shape

\section{Introduction}

Grain size is a fundamental property of the soils and sediments, which can provide information on their origin with particular regard to transport dynamics, deposition and post-depositional alterations of sedimentary mineral particles. These properties can be deciphered from the particle size distribution. Generally, the sedimentary deposits may contain a wide range of particle sizes from boulder fraction to the smallest size, clay and colloid (McCAvE, N. and SyvitSKI, J.P.M. 1991). In most landscape development system, the particle size distribution of the constituent sediments reflects the morphological characteristics associated with the physical processes of development processes (Switzer, A.D. 2013). The importance of the particle size distribution lies in resembling the physico-chemical properties of materials (e.g., particle size) which are determined by the power and capacity of the transporting agent. Furthermore, sediments and soils have

\footnotetext{
${ }^{1}$ Eötvös Loránd University, Faculty of Science, Department of Environmental and Landscape Geography, Pázmány Péter sétány 1/c. H-1117 Budapest, Hungary; Geographical Institute, Research Centre of Astronomy and Earth Science. Budaörsi út 45. H-1112 Budapest, Hungary. E-mail: gresina.fruzsina@csfk.mta.hu
} 
specific features that depend on their particle size distribution, e.g., hydraulic properties (porosity, mass density, water content, water retention), thermal conductivity, and specific surface area (CAmpbell, G.S. and Shiozawa, S. 1992; Udvardi, B. et al. 2017).

Before the recent paradigm shift (BLotT, S.J. and PYE, K. 2006; ÚJVÁRI, G. et al. 2016; VARGA, Gy. et al. 2019a) particle size distribution was measured by sieving and sedimentation method. This can be expressed as a percentage by size class, as a fraction of total dry grains by volume or weight. The relatively new and fast-spreading of laser diffraction methods raises the question of how similar are the data by laser particle sizing and classical techniques. Numerous studies were published to discuss the difference between the results of new particle size determination methods and conventional approaches (e.g. Syvitski, J.P.M. et al. 1991; Konert, M. and VAndenberghe, J. 1997; Beuselinck, L. et al. 1998; di Stefano, C. et al. 2010; Centeri, Cs. et al. 2015a, b). The object of these studies is generally to determine and compare the clay fraction with the results obtained by the different methods. Simultaneously, the question may arise, where to draw the boundary of the clay fraction. The limits of the ranges may change during sample preparation (aggregates disintegration, removal of grain coatings by chemical pretreatment). From these studies, it can be concluded that the clay content of a sample depends on its clay mineral composition and properties, including particle shape characteristics.

\section{Materials and methods}

Recent soil and sediment samples were investigated in the present study. Various methods analysed the particle size distribution of Gleysol horizon B, haplic Luvisol horizon $\mathrm{B}_{\mathrm{t}}$ and podzolic Luvisol horizon $\mathrm{C}$. Furthermore, loess and sandy loess were included in the particle size studies. Samples were collected from Hungarian locations with special attention to the representation of samples with generally different granulometric character (from clayey to more sandy texture). Loess and sandy loess were collected from Köszárhegy, Podzolic Luvisol and haplic Luvisol samples were taken from Sopron, lastly, Gleysoil was collected from Ceglédbercel (Figure 1). Table 1. shows the applied methods which performed on the samples.

Large aggregates and rock-fragments were separated using a $2 \mathrm{~mm}$ sieve. Before the first set of measurements, the samples were only pretreated with sodium pyrophosphate (5\%) to disintegrate aggregates into individual particles. During the next series of measurements, the organic matter and $\mathrm{CaCO}_{3}$ coatings were also removed from the test samples by using hydrogen peroxide $(30 \%)$ and hydrogen chloride $(10 \%)$. Pipette method and laser diffraction were used during the study.

The pipette method is based on the change in density over time calculated from suspension settling time $(t)$ and depth $(z)$, which gives all the grains in the original concentration $\left(V_{s} \leq z / t\right)$. This assumes that the particles settle independently, there is no flocculation, and the temperature is constant. The solid material content of the test sample is determined by evaporation and mass measurement. A correction value is used to subtract the dissolved salts of the sample. The cumulative curve of the mass of sedimentation fractions can be determined by the Stokes law:

$$
V s=\Delta \rho \cdot g \cdot d^{2} / 18 \mu,
$$

where $\Delta \rho$ is the density difference between the liquid and the particles, $d$ is the diameter of the particles, $\mu$ is the molecular viscosity, and $g$ is acceleration by gravity (McCAVE, N. and SYviTSKI, J.P.M. 1991). The pipette method expresses the number of particle size classes by weight.

During the procedure of pipette method, 25-25 g of material was weighed together with 1 litre of distilled water in the settling cylinders (5 sets). Larger particles were trapped using a $250 \mu \mathrm{m}$ sieve. Three (Podzolic Luvisol horizon $C$, haplic Luvisol horizon $B_{t^{\prime}}$ and loess) of the five samples were used for the evaluation of the pipette method. 


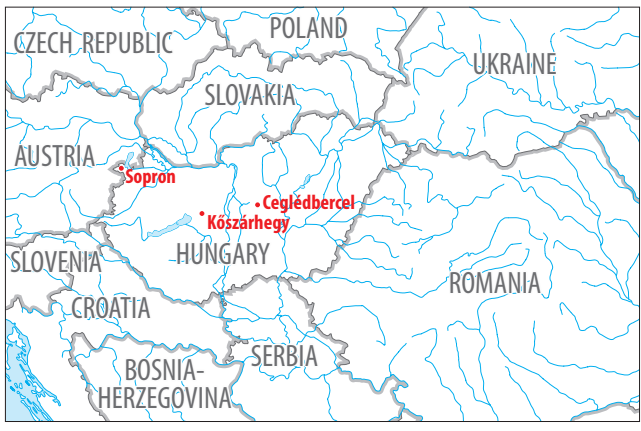

Fig. 1. Map of the sampling areas

\begin{tabular}{l|c|c}
\multicolumn{2}{c}{$\begin{array}{c}\text { Table 1. Applyed grain size analysis methods in the } \\
\text { case of different samples }\end{array}$} \\
\cline { 2 - 3 } \multicolumn{1}{c}{ Samples } & Measurements by \\
\cline { 2 - 3 } & $\begin{array}{c}\text { pipette } \\
\text { method }\end{array}$ & $\begin{array}{c}\text { laser } \\
\text { diffraction }\end{array}$ \\
\hline $\begin{array}{l}\text { Loess } \\
\text { Luvisol }\end{array}$ & 5 repetiton \\
$\begin{array}{l}\text { Podzolic Luvisol } \\
\text { Sandy loess } \\
\text { Gleysol }\end{array}$ & $\varnothing$ & 5 repetition \\
\end{tabular}

Deviations from the results calculated using the theoretical Stokes law (suspecting spherical particle shapes) can be expected if the particles are irregularly shaped, as most clay particles have a flat, lamellar shape. The non-spherical particles settle with their maximum cross-section projection perpendicular to the settling direction. Consequently, this situation increases the expected tensile strength of the particle and reduces the settling rate. The particle shape affects the results, as an overestimation of the so-called fine fraction (Di Stefano, C. et al. 2010). The pipette method has more other drawbacks: it is time-consuming, highly dependent on laboratory technique and operator error (Syvitski, J.P.M. et al. 1991). It requires a large volume of samples (at least 20-25 g) for analysis. Hence, the speed of the method is not sufficient to accurately analyse large numbers of samples (BEUSELINCK, L. et al. 1998).
The laser diffraction particle size analysis is based on the interaction of laser light and the particles, as reflection, refraction, absorption and diffraction of light (caused by the particle) result in a specific light scatter pattern depending on particle size. The angle and intensity of scattered light are transformed into particle size distribution by different optical theories. The traditional laser diffraction analysers are based solely on the principle that particles of a given size diffract the light at a given angle. The angle of diffraction increases with decreasing particle size (McCave, N. and Syvitski, J.P.M. 1991).

Generally, two optical models can be used to calculate particle size from the light intensity: Fraunhofer and Mie theories (GEE, G.W. and OR, D. 2002). Fraunhofer diffraction calculates only by the angle of light diffraction (DE BoER, G.B.J. et al. 1987), while Mie theory also takes into account the events of light absorption and refraction during particle size determination (EsHeL, G. et al. 2004). Both theories assume that the particles are spherical. Thus, the particle diameter obtained from the laser diffraction is equivalent to the sphere that gives the same diffraction as the particle (di Stefano, C. et al. 2010). The sphere is the only shape which crosssectional diameter is constant, regardless of the angle at which it is viewed.

The problem is that natural particles have different cross-sections in all directions. Thus, the cross-sectional area of a non-spherical particle is larger than that of a sphere having the same volume as the particle. It places this tested particle in a larger size range than can be inferred from its apparent radius. Thus, there is a shift in size distribution towards coarser fractions (EsHel, G. et al. 2004). The laser diffraction gives the particle size distribution as a percentage by volume. The laser diffraction devices which originate from different manufacturers usually differ from each other. These differences based on the laser systems, the number of detectors and the measuring range (Table 2).

During the refraction and absorption adjustments, the Mie theory was applied to the 
Table 2. Measuring properties of laser diffraction devices

\begin{tabular}{l|l|c|c|c|c}
\hline Manufacturer & \multicolumn{1}{|c|}{ Device } & $\begin{array}{c}\text { Measuring } \\
\text { range, } \mu \mathrm{m}\end{array}$ & $\begin{array}{c}\text { Number of } \\
\text { sensors }\end{array}$ & $\begin{array}{c}\text { Optical } \\
\text { method }\end{array}$ & \multicolumn{1}{|c|}{ Type of laser } \\
\hline Horiba Ltd. & $\begin{array}{l}\text { LA-950 Laser Particle } \\
\text { Size Analyser }\end{array}$ & $0.01-3,000$ & n.d. & Mie & $\begin{array}{l}650 \mathrm{~nm} \text { (red), } \\
405 \mathrm{~nm} \text { (blue) }\end{array}$ \\
Fritsch GmbH. & Analysette 22 & $0.01-2,100$ & 57 & $\begin{array}{l}\text { Fraunhofer, } \\
\text { Mie }\end{array}$ & $\begin{array}{l}532 \mathrm{~nm} \text { (green), } \\
850 \mathrm{~nm} \text { (infrared) }\end{array}$ \\
\hline
\end{tabular}

n.d. = No data.

Horiba Partica LA 950 V2 with a refractive index of 1.45 and a light absorption value of 0.1 (according to the recommendations of VARGA, Gy. et al. 2019b). The other device which was used during the study was the Fritsch Analysette 22 Microtec Plus. Quartz refractive index of 1.54 and light absorption value of 0.1 was selected in the measurements settings (additionally, the Fraunhofer settings were also applied during the Fritsch measurements). Measurements were made in wet dispersion in the case of both devices (RI of water [1.33] was applied).

The shape of the particles can be characterised by various properties, e.g., shape, roundness, and sphericity of the irregularity (Blott, S.J. and Pye, K. 2008). The shape of a particle can be described by its three-dimensional characteristics, which are defined by the ratio of length, thickness, and width (SNEED, E.D. and Folk, R.L. 1958). Convexity describes how closely the shape of a given particle approximates the form of a real sphere (in two dimensions, this property is called circularity). If the surface of a particle has significant depressions (concavity) and protrusions (convexity), its shape can be sensed irregular (BLOtT, S.J. and PyE, K. 2008). The method was applied to each of the five samples in order to obtain information on the shape of the particles examined.

The shape information of the tested sediments and soils was provided by the Malvern Morphologi G3-ID automated static image analyser. Recently, morphological characterization of grains is a dynamically developing method for investigating various sediments (Moss, A.J. 1966; Rogers, C.D.F. and Smalley, I.J. 1993; VARGA, Gy. et al. 2018;
VArgA, Gy. and Roettig, C.-B. 2018; Király, Cs. et al. 2019). The number of analysed grains was not sufficient for robust statistical analysis. This problem has been successfully overcome with automatized systems (Cox, M.R. and Budhu, M. 2008). Image analysis provides direct observational data of particle size, and due to the automatic measurement technique, a large number of particles are characterised allowing us a more robust and objective granulometric description of particles compared to manual microscopic approaches (VARGA, Gy. et al. 2018). Image analysis-based measurements were organised into a number-based database, which can be transformed into a volumetric database as well. All of the particles have their identity number (ID). The applied greyscale intensity threshold was $0-45$ with $20 \times$ objective. The shape parameters were determined automatically. Circularity and aspect ratio were analysed in this study as attributes of the individual particles. Aspect ratio is the ratio of width and length, and circularity parameter of a particle describes the proportional relationship between the circumference of a circle equal to the object's projected area and perimeter.

Particle size ranges of pipette method were also used in the case of laser diffraction to compare the results of the two approaches adequately. However, during comparison of the results of the two laser diffraction devices, the three distribution curves (Fritsch Analysette 22 Mie and Fraunhofer models, Horiba Partica LA 950 V2 Mie theory) with the original grain size bin allocations were plotted together. For samples that only have undergone laser diffraction measurements, 
a different representation was used. This definition applies to sandy loess and B horizon of Gleysol. The same chart shows the results of untreated and pretreated samples per device. Bar chart and connected dot chart types were used to display data. Beyond the visual evaluation of the graphs, the results were compared by performing the linear regression analyses $(n=13)$.

\section{Results}

\section{Comparison of laser diffraction and pipette results}

For the untreated sample of loess (Figure 2, a), the $<2 \mu \mathrm{m}$ fraction can be characterised by the highest volumetric proportion compared to other ranges. This trend is valid for all four distributions. The results of the pipette method were not significantly different from the laser diffraction. For the pretreated samples (Figure 2, b), the proportion of the $<2 \mu \mathrm{m}$ range decreased and the volumetric contribution of silt fractions (10-20 $\mu \mathrm{m}$ and $20-50 \mu \mathrm{m})$, increased in the case of all four methods.

Based on Figure 3 (a), and Figure 3 (b), it can be stated that the hydrochloric acid and hydrogen peroxide pretreatment resulted in a more even distribution in all three cases. Typical loess distribution was obtained with a minor deviation for the smallest fractions. In the case of untreated (Figure 3, a) elemental particles, there are some differences in the distribution curves, especially in the sub-micron fraction. It is also important to note that the difference between Fraunhofer diffraction and Mie theory was also apparent, although the same instrument measured it. The Fraunhofer optical model does not show a secondary maximum in the untreated sample, in contrast to the Mie theory. Besides, the result obtained by the Horiba instrument shows the highest secondary peak for the same sample. So, the difference was reflected in the results obtained by the same optical model, which was measured with different manufacturers' equipment. In the case of the pretreated sample (Figure 3, b) the difference is reduced, partly because of the larger particle size ranges converge. The regression coefficient between the two devices was above $0.94\left(\mathrm{R}_{\text {untreated }}^{2}=0.93\right)$.

In the case of Luvisol, similarly to loess, pretreated (Figure 2, d and Figure 3, d) samples show a more uniform distribution than the untreated ones (Figure 2, c and Figure 3, c). The ratio of the $<2 \mu \mathrm{m}$ particles was materially reduced by hydrogen peroxide pretreatment, especially in the case of Horiba particle size analyser, where the volume percentage of the $<0.5 \mu \mathrm{m}$ fraction decreased from 12 to 0 per cent. Simultaneously, the proportion of larger particle increased, especially for 31-63 $\mu \mathrm{m}$ and $63-125 \mu \mathrm{m}$. The regression coefficient showed a higher value for pretreated samples of laser diffraction devices $\left(\mathrm{R}^{2}\right.$ untreated $=0.72 ; \mathrm{R}_{\text {pretreated }}^{2}=0.89$ ).

The third sample, the horizon C of Podzolic Luvisol, was subjected to pretreatment combined with the pipette method. The comparison was made by using two series of pipette measurements. As a result, the size of the particles was influenced by the duration of pretreatment (Figure 2, g). The mass proportions of the finest and the coarsest size fractions showed a significant decreasing trend as a function of pretreatment time. A general increase of silt-sized particles could be detected after longer pretreatment. The parallel measurements size distribution with longer pretreatment time was much more similar to the results obtained by laser diffraction. That is why it was used to compare the different method's grain size distributions.

In the case of Podzolic Luvisol sample, there were substantial differences between the untreated and pretreated samples. Figure 2 (e) shows that the distributions of untreated samples are following two types of curves. The Mie and Fraunhofer results of Fritsch device are almost identical. However, they differ from the Horiba values and the results of the pipette method. The former has detected a larger ratio in size range of $<20 \mu \mathrm{m}$. The latter shows a steady increase in diameter towards the larger particles. As an effect of the pretreatment (Figure 2, f), the size data which were obtained by Fritsch device got closer to the 

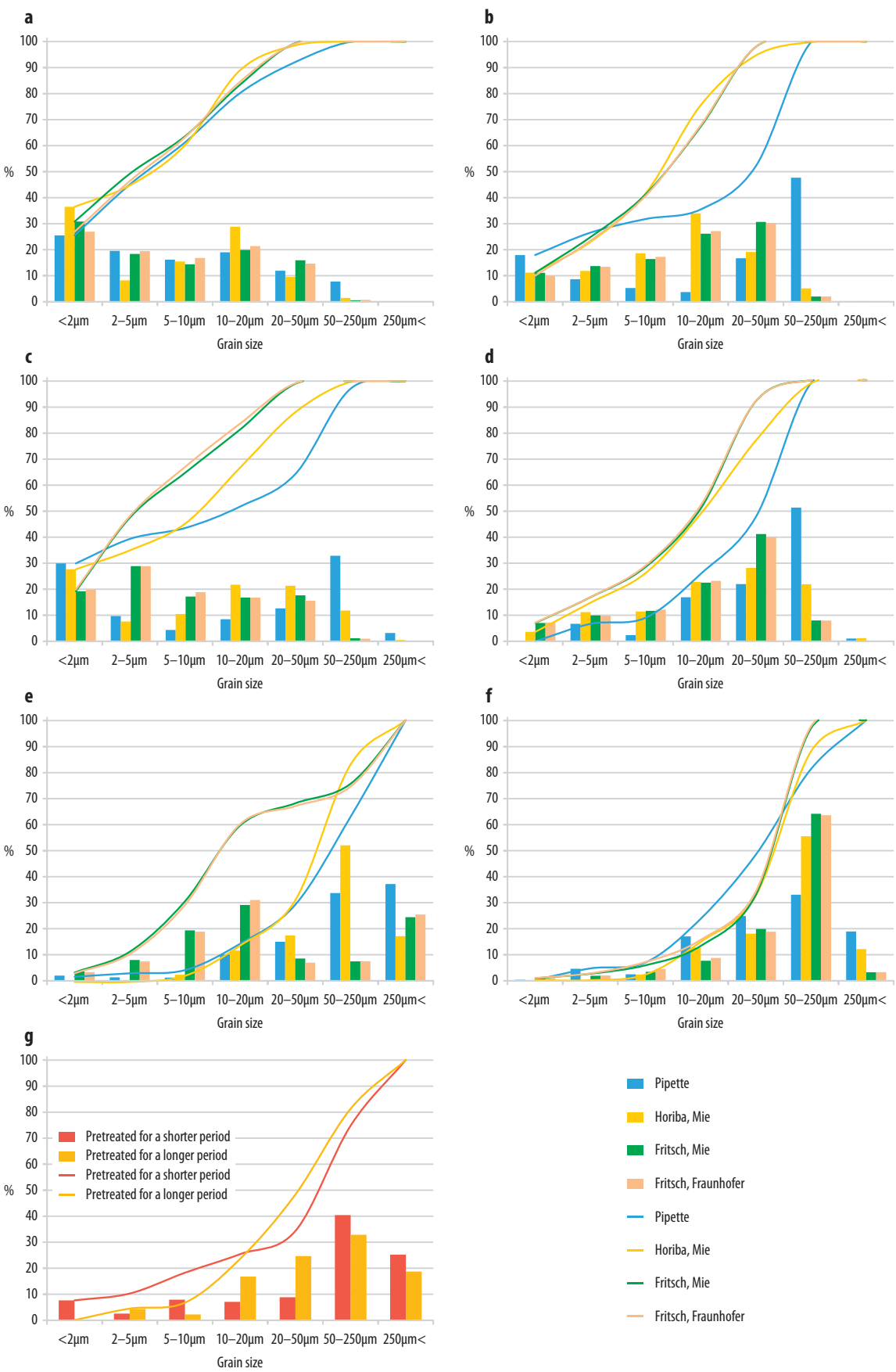

Fig. 2. Particle size distributions of the samples as regards the results obtained by the laser diffraction method and pipette - Pipette $=$ percentage by weight; Laser diffraction $=$ percentage by volume; $\mathrm{a}=$ Loess, untreated; $\mathrm{b}=$ Loess, pretreated; $\mathrm{c}=$ Luvisol, untreated; $\mathrm{d}=$ Luvisol, pretreated; $\mathrm{e}=$ Podzoic Luvisol, untreated; $\mathrm{f}=$ Podzolic Luvisol, pretreated; $\mathrm{g}$ = Podzolic Luvisol, pretreated by two methods 

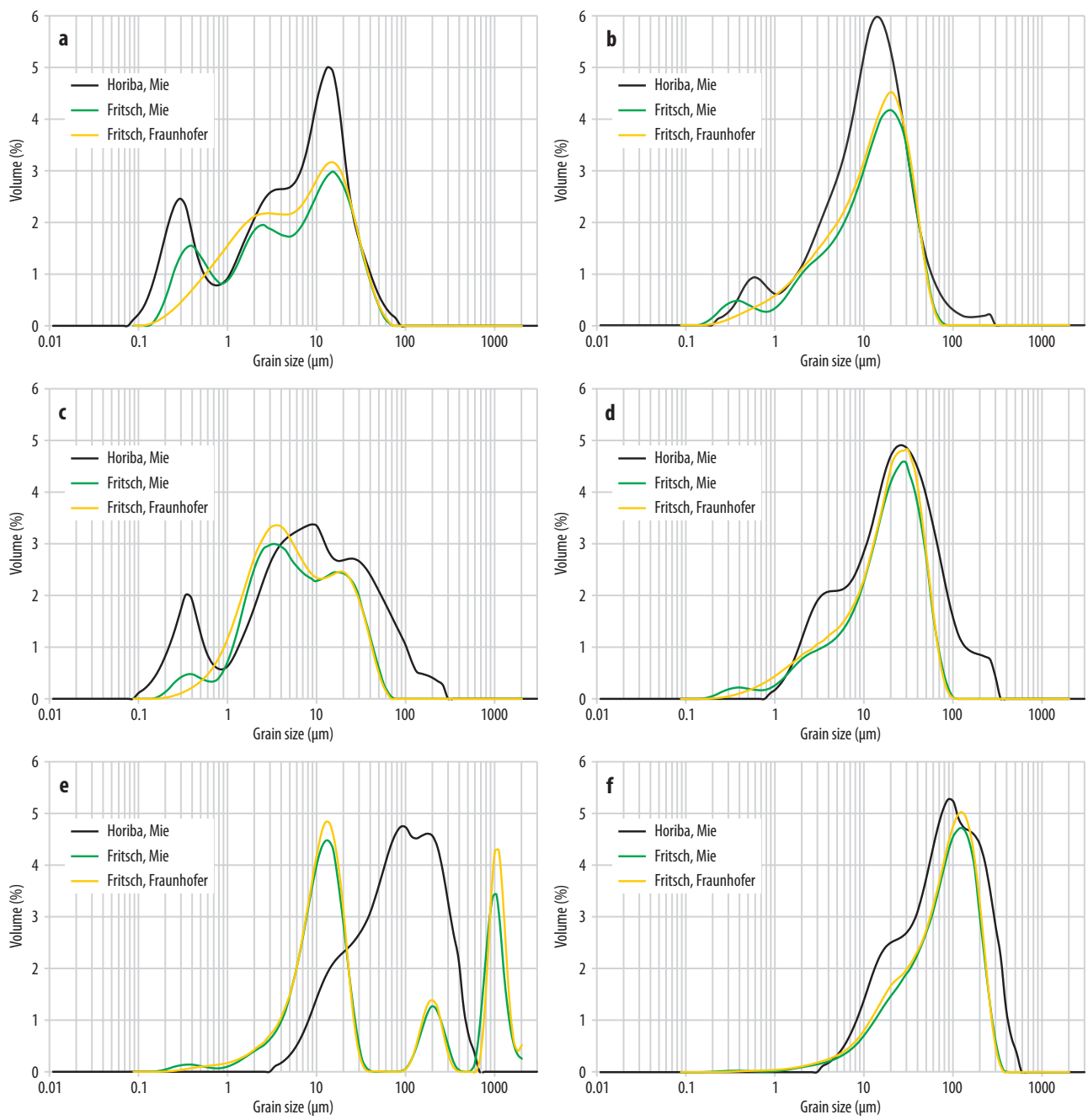

Fig. 3. Particle size distributions of samples based on laser diffraction results. Comparison of different instruments. $-\mathrm{a}=$ Loess, untreated; $\mathrm{b}=$ Loess, pretreated; $\mathrm{c}=$ Luvisol, untreated; $\mathrm{d}=$ Luvisol, pretreated; $\mathrm{e}=$ Podzolic Luvisol, untreated; $\mathrm{f}=$ Podzolic Luvisol, pretreated

Horiba's and pipette method's results. The value of the $\mathrm{R}^{2}$ increased to 0.93 between the two laser diffraction devices obtained by the Mie theory $\left(\mathrm{R}^{2}\right.$ untreated $\left.=0.0\right)$. In the case of the Fritsch instrument (Figure 3, e), there are two secondary maxima in the Fraunhofer as well as in the Mie distributions. The maximum is at $14 \mu \mathrm{m}$, and the other two additional peaks are at $200 \mu \mathrm{m}$ and $1,000 \mu \mathrm{m}$. In the case of the
Horiba instrument, the distribution is much smoother, since the smallest value of the distribution is $\sim 3.4 \mu \mathrm{m}$, while the maximum is at $\sim 100 \mu \mathrm{m}$. All in all, the results of the two devices are entirely different. The pretreatment, however, resulted in much more uniform distributions of Podzolic Luvisol samples (Figure 3, f). Similar significant differences were reported by VARGA, Gy. et al. (2019b). 
Comparison of different optical models of laser diffractometry and effects of sample pretreatments

Results of laser diffraction measurements of sandy loess samples are presented in Figure 4 (a) and (b). Volumetric proportions of $<10$ $\mu \mathrm{m}$ fractions of treated and untreated Horiba results were significantly different from each other; the pretreatment resulted in a substantial decrease in this range, but, the two secondary maxima remained the same. The Mie results of Fritsch device shows a similar tendency: as a result of the pretreatment (Figure $4, \mathrm{~b}$ ), the percentage of $<10 \mu \mathrm{m}$ fraction has decreased. The value of the regression coefficient increased as a result of pretreatment as measured by the Horiba and Fritsch instrument using Mie theory: $\mathrm{R}_{\text {treated }}^{2}=0.87$; while $R_{\text {untreated }}^{2}=0.43$. It is worth noting that results calculated by using the Fraunhofer theory were profound than the grain sizes of Mie settings. However, the three laser diffraction curves are moving together in the ranges above $100 \mu \mathrm{m}$. The result of the horizon B of Gleysol obtained by the Horiba laser diffraction particle size analyser shows typical characteristics of the tendency in the literature (Figure 4, c), that the proportion of smaller particle size diameters increases after pretreatment procedures (DI STEFANO, C. et al. 2010). The same tendency can be observed in the case of Fritsch device (Figure 4, d), with only a slight shift towards smaller particle size ranges. In the case of the Fraunhofer optical model, it should be emphasised that the pretreatment did not perform the expected result, as no secondary maximum was achieved after the pretreatment. How-
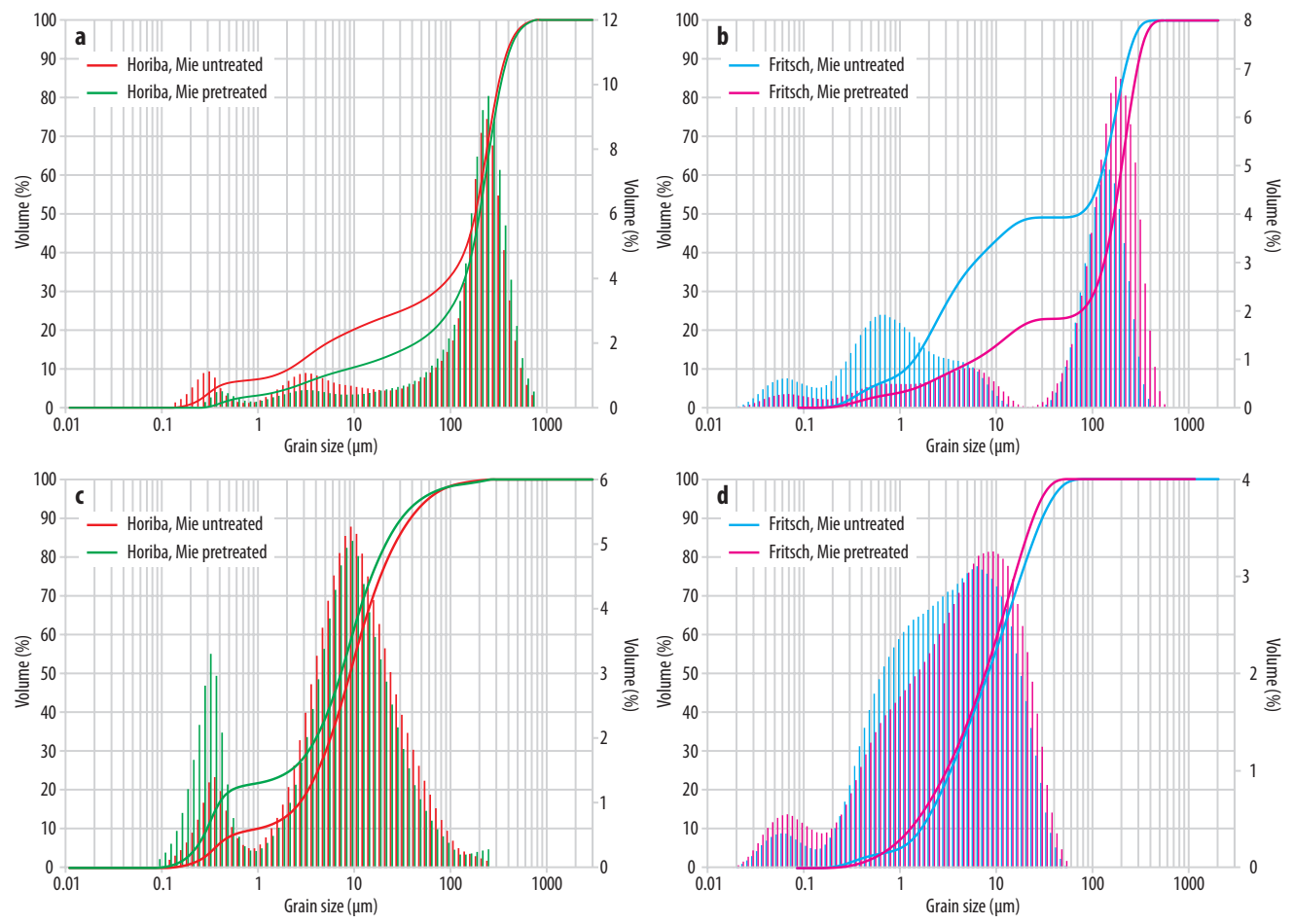

Fig. 4. Particle size distributions of samples based on laser diffraction results. Comparison of different instruments. - $\mathrm{a}$ = Sandy loess, Horiba Partica LA 950 V2; b = Sandy loess, Fritsch Analysette 22; c = Gleysol, Horiba Partica LA 950 V2; d = Gleysol, Fritsch Analysette 22 
ever, the value of the regression coefficient decreased in this case as a result of pretreatment between Horiba and Fritsch instrument results: $R_{\text {treated }}^{2}=0.63$ and $R_{\text {untreated }}^{2}=0.8$.

Table 3 summarises the average values of two Malvern Morphologi G3-ID particle shape parameters (circularity and aspect ratio). The size-dependence of these properties were also tested; the circularity values decrease from the smaller fractions towards the larger sizes (Figure 5, a-e), but larger grains typically have high aspect ratio, although their circularity parameter is relatively low.

Table 3. Comparison of the volume-weighted mean shape properties of the untreated tested samples, Malvern Morphologi G3-ID

\begin{tabular}{l|c|c}
\hline \multicolumn{1}{c|}{ Samples } & Circularity & $\begin{array}{c}\text { Aspect } \\
\text { ratio }\end{array}$ \\
\hline Loess & 0.921 & 0.841 \\
Luvisol horizon $\mathrm{B}_{\mathrm{t}}$ & 0.960 & 0.879 \\
Podzolic Luvisol horizon C & 0.884 & 0.821 \\
Sandy loess & 0.933 & 0.844 \\
Gleysol horizon B & 0.944 & 0.885 \\
\hline
\end{tabular}

\section{Discussion}

By the spread of new techniques, several research groups have tried to compare and match the results obtained with different techniques. These studies reported the clear uncertainties of determination of the clay and fine silt fractions, and unknown particle morphology was proposed to be a possible cause of the mismatches (KonerT, M. and VAndenberghe, J. 1997; BeAuselinck, L. et al. 1998; Pieri, L. et al. 2006; di Stefano, C. et al. 2010).

The results of this paper only partially reflect the trends found in the literature. According to Di Stefano, C. et al. (2010), the laser diffraction underestimates the proportion of clay fraction compared to the pipette method, however, this tendency is only partially true for our results since the ratios of different size fractions (including the claysized particles) were changed (primarily) due to the hydrochloric acid and hydrogen peroxide pretreatments. In the case of loess, the laser diffraction detected a higher proportion of the $<2 \mu \mathrm{m}$ fraction in the untreated sample than the pipette method. However, this phenomenon reversed as a result of carbonate removal. The smallest particles of Luvisol has practically disappeared according to pipette method after the pretreatment $(<2 \mu \mathrm{m}$ fraction of the untreated sample was $\sim 29.8 \%$ ). The case of Podzolic Luvisol was different; the emphasis was on the $>10 \mu \mathrm{m}$ ranges and their changes. These phenomena are because the investigated materials had different mineral composition and organic content. The disintegration of aggregates and removal of grain coatings which were responsible for the larger grain size these established smaller particles, even nanometres in size which can no longer be detected by these methods. So the emphasis shifted towards the relatively larger size ranges.

The particle size distribution measured by laser diffraction particle size analyser does not match the values determined by classical methods, which has several causes. Laser diffraction gives a percentage by volume, whereas conventional methods (sedimentation, sieving) give a percentage by weight. The result of laser diffraction is generally independent of the density of the particles, whereas the pipette method is based on the change of density over time (SYvitski, J.P.M. et al. 1991). These few differences are enough to give different results for the same sample.

The literature on the results of laser diffraction instruments is controversial, according to di Stefano, C. et al. (2010) there is no significant difference between the cumulative distribution curves determined by the two optical theories. While BAYweL, L.P. and Jones, A.L. (1981) and de Boer, G.B.J. et al. (1987) report significant differences in the smaller grain size ranges. Based on our study, results of Fraunhofer diffraction are significantly different from Mie theory, because it can detect much lower volume percentages of finer particles. This theory assumes that the laser beam is parallel, and the sensors are at a great distance relative to 

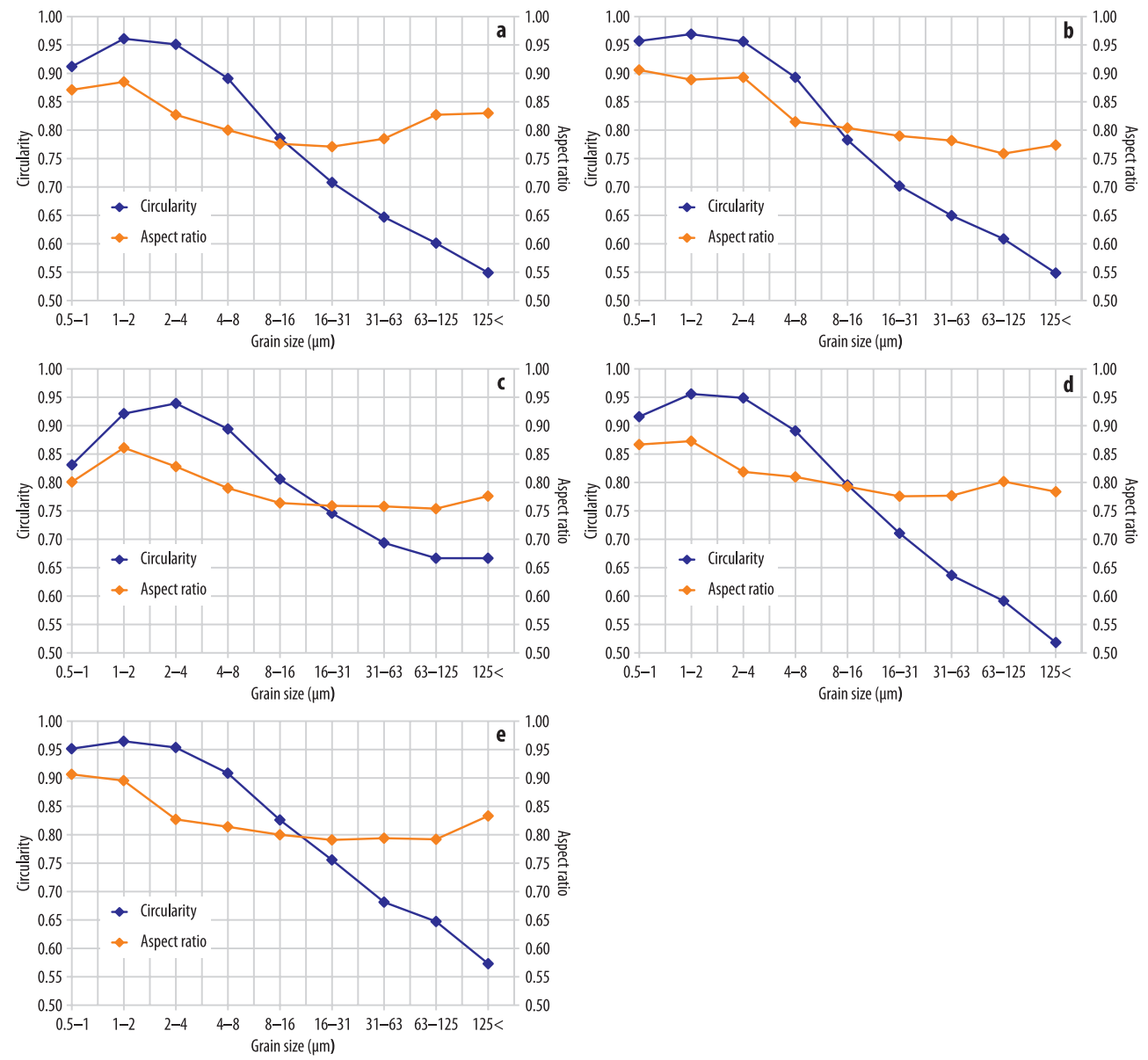

Fig. 5. Comparison of the volume weighted mean shape properties of the size ranges of the untreated tested samples, Malvern Morphologi G3-ID. $-\mathrm{a}=$ Loess; $\mathrm{b}=$ Luvisol; $\mathrm{c}=$ Podzolic Luvisol; $\mathrm{d}$ = Gleysol; $\mathrm{e}=$ Sandy loess

the size of the diffracted particle (Loizeau, J.L. et al. 1994). If the particles are larger than the wavelength of the light, the interaction can be interpreted as diffraction (DE BOER, G.B.J. et al. 1987). The Fraunhofer theory becomes inapplicable when the particle diameter approaches the wavelength of light. As the refraction of the grain falls within this size range, this principle is no longer applicable (LoizeAu, J.L. et al. 1994). Therefore, any comparison of fine-grained (clay- and fine silt-sized) fractions measured by different laser and traditional methods will provide different results for each sedimentary sample (VARGA, Gy. et al. 2019b).

According to VARGA, Gy. et al. (2019b), if only one laser diffraction device is used with the same optical settings for all samples from the investigated profile, the significant relative changes of measured data and calculated values will reveal the general trends. Nevertheless, absolute values can only be compared if the same optical settings and the same devices were used. Unfortunately, 
a large proportion of research papers still do not discuss the applied laser diffraction measurement settings properly, and the specification of the applied optical approach and complex refractive index are the most commonly missing pieces of information (VARGa, Gy.et al. 2019b).

All in all, Horiba and Fritsch grain size results of samples with a higher volumetric proportion of larger particles (sandy loess, Podzolic Luvisol, loess) were more comparable than the results of more clayey samples. This may be because the coarse-grained samples are characterized by a higher proportion of more spherical particles than the clayey Gleysol with more irregular mineral grains. The shape of sand-sized grains is more similar to a sphere than the particles of smaller size ranges (Polakowski, C. et al. 2014). Therefore, the methods were more compatible with sandy samples. The results of particle shape analysis by optical microscope were also contradictory since particle circularity of smaller size ranges $(<4 \mu \mathrm{m})$ were closer to 1 than the larger grains. This was contrary to the trend found in the literature. The reason for this was probably due to the presence of adhesives $\left(\mathrm{CaCO}_{3^{\prime}}\right.$ organic matter), which formed aggregates in the sample, thus were distorting the shape distribution. The number of pixels decreases with grain size. The smaller particles have smaller area covered by pixels, which can lead to simplified shape properties compared to larger grains. In the case of Luvisol the circularity property was the highest among the other samples. This sample had a relatively large amount of small particles $(0.5-2.0 \mu \mathrm{m})$ which did not led to proper shape properties over the whole sample. Consequently, analysis by separating aggregates is warranted. However, the device does not show a three-dimensional image of the shape of the particles. The third dimension of particles cannot be accurately determined by automated static image analysis as the orientation of individual particles is not random, they are facing into the CCD-camera with their largest surface area (VARGA, Gy. et al. 2018).

\section{Conclusions}

Nowadays, the laser diffraction technique is one of the most advanced methods for determining particle size distribution. In contrast to the classical techniques, these measurements are faster, more reproducible, and need a relatively small amount of material.

Depending on the purpose of the measurements, the question of the need for chemical pretreatment of samples has to be taken into account too. It can be stated that it greatly influences the obtained results. It may be questionable to what extent it is advisable to use hydrochloric acid pretreatment for loess since a significant part of the sediment is composed of carbonate. Is it worth removing carbonates completely if they build up real grains?

If the purpose of the measurements with different devices is data harmonization, it is advisable to use the same unit of measurement. It is not advisable to represent the measurement methods together in various dimensions, treating them in the same plane since they measure different properties of the particles. When combining data from different laser diffraction devices, special care should be taken to ensure that measurements are made with the same optical adjustment, with particular reference to the value of the refractive index. In the case of measurements of the smallest grain fractions, the obtained results must be treated with caution. The laser diffraction devices which were used in this study have different structure, the results obtained by them may not be the same; dual laser systems developed by different manufacturers do not operate in the same wavelength range, however, according to VARGA, Gy. et al. (2019b), the wavelength of built-in laser(s) do(es) not have an effect on the results. It may be advisable to include a device capable of measuring in the submicron range (photon correlation spectroscopy). An inter-laboratory comparison could help for optimizing techniques for different sediment types as well as set new standards in particle size determination methods. Also, 
it is worth getting some information about the mineral composition and shape of the particles (optical microscopy, electron microscopy), which can help to explain certain phenomena and differences between the distribution curves. When using an optical microscope, it is worth considering the irregularity of the particles as a function of the aspect ratio of the particles rather than the circularity parameter.

Even before the expansion of the laser diffraction technique, the standardisation of particle size distribution was difficult. For more than two decades, Syvitski, J.P.M. et al. (1991) have stated that although there are many methods for determining particle size, none in sedimentology and geomorphology can be accepted as an uncompromising procedure.

In the present study, the particle size distributions were compared, however, beyond these methods there are more complex analyses which can be used depending on what property is desirable (simple statistical methods, ratio-based indicators, mathematical-statistical methods). In geomorphology and sedimentology, the determination of particle size distribution is rarely the ultimate goal. The objectives are to determine the evolution of surface forms, and the conditions of transport as well as the deposition of the grains. One of its key components is the determination of particle size distribution (SwITZER, A.D. 2013). Interpretation of granulometric (particle size and particle shape) data enables understanding and reconstruction of sedimentation environments and processes controlling surface evolution.

Acknowledgements: The study is supported by the ÚNKP-19-3 New National Excellence Program of the Ministry for Innovation and Technology. Support of the National Research, Development and Innovation Office NKFIH K120620 and KH130337 are gratefully acknowledged.

\section{REFERENCES}

BAYVEL, L.P. and Jones, A.R. 1981. Electromagnetic scattering and its applications. Applied Science, London, Englewood N.J.
Beuselinck, L., Govers, G., Poesen, J., Degraer, G. and Froyen, L. 1998. Grain-size analysis by laser diffractometry: comparison with the sieve-pipette method. Catena 32. (3-4): 193-208.

BLOTt, S.J. and PYE, K. 2008. Particle shape: a review and new methods of characterisation and classification. Sedimentology 55. (1): 31-63.

Campbell, G.S. and Shiozawa, S. 1992. Prediction of hydraulic properties of soils using particle-size distribution and bulk density data. In Indirect methods for estimating the hydraulic properties of unsaturated soils. Eds.: van Genuchten, M.T., LeiJ, F.J. and Lund, L.J., Riverside, University of California, 317-328.

Centeri, Cs., Jakab, G., Szabó, Sz., Farsang, A., Barta, K., Szalai, Z. and Bíró, Zs. 2015a. Comparison of particle-size analysing laboratory methods. Environmental Engineering and Management Journal 14. (5): 1125-1135.

Centeri, Cs., Szalai, Z., Jakab, G., Barta, K., Farsang, A., Szabó, Sz. and Bíró, Zs. 2015b. Soil erodibility calculations based on different particle size distribution measurements. Hungarian Geographical Bulletin 64. (1): 17-23.

Cox, M.R. and Budhu, M. 2008. A practical approach to grain shape quantification. Engineering Geology 96. 1-16.

De Boer, G.B.J., de Weerd, C., Thoenes, D. and Goossens, H.W.J. 1987. Laser diffraction spectrometry: Fraunhofer versus Mie scattering. Particle Characterisation 4. (1-4): 14-19.

di Stefano, C., Ferro, V. and Mirabile, S. 2010. Comparison between grain-size analyses using laser diffraction and sedimentation methods. Biosystem Engineering 106. (2): 205-215.

Eshel, G., Levy, G.J., Mingelgrin, U. and Singer, M.J. 2004. Critical evaluation of use of laser diffraction for particle-size distribution analyses. Soil Science Society of America 68. 736-743.

GeE, G.W. and OR, D. 2002. Particle size analysis. In Soil science society of America book series, Vol. 5. Methods of soil analysis, Part 4. Physical methods. Eds.: DANE, J.H. and Topp, G.C., Madison, WI, 255-293.

Király, Cs., Falus, Gy., Gresina, F., Jakab, G., Szalai, Z., and VARGA, Gr. 2019. Granulometric properties of particles in Upper Miocene sandstones from thin sections, Szolnok Formation, Hungary. Hungarian Geographical Bulletin 68. (4): 341-353.

Konert, M. and Vandenberghe, J. 1997. Comparison of laser grain size analysis with pipette and sieve analysis: a solution for the underestimation of the clay fraction. Sedimentology 44. 523-535.

Loizeau, J.L., Arbouille, D., Santiago, S. and Vernet, J.P. 1994. Evaluation of a wide range laser diffraction grain size analyser for use with sediments. Sedimentology 41. 353-361.

McCave, N. and Syvitski, J.P.M. 1991. Principles and methods of geological particle size analysis. 
In Principles, methods and application of particle size analyses. Ed.: Syvitski, J.P.M., Cambridge, Cambridge University Press, 3-22.

Moss, A.J. 1966. Origin, shaping and significance of quartz sand grains. Journal of the Geological Society of Australia 13. 97-136.

Pieri, L., Bittelli, M. and Pisa Rossi, P. 2006. Laser diffraction, transmission electron microscopy and image analysis to evaluate a bimodal Gaussian model for particle size distribution in soils. Geoderma 135. 118-132.

Polakowski, C., Sochan, A., Bieganowsky, A., Ryzak, M., FöLDÉNYI, R. and Tóth, J. 2014. Influence of the sand particle shape on particle size distribution measured by laser diffraction method. International Agrophysics 28. (2): 195-200.

Rogers, C.D.F. and Smalley, I.J. 1993. The shape of loess particles. Naturwissenschaften 80. 461-462.

SNeed, E.D. and Folk, R.L. 1958. Pebbles in Lower Colorado River, Texas: a study in particle morphogenesis. Journal of Geology 66. (2): 114-150.

Switzer, A.D. 2013. Measuring and analysing particle size in a geomorphic context. In Treatise on Geomorphology 14. Methods in geomorphology. Eds.: Shroder, J., Switzer, A.D. and Kennedy, D.M., San Diego, CA, Academic Press, 224-242.

Syvitski, J.P.M., Leblanc, K.W. and Asprey, K.W. 1991. Interlaboratory, instrument calibration experiment. In Principles, methods and application of particle size analyses. Ed.: Syvitski, J.P.M., Cambridge, Cambridge University Press, 174-193.
ÚJvÁRI, G., KoK, J.F., VARGA, Gy. and Kovács, J. 2016. The physics of wind-blown loess: Implications for grain size proxy interpretations in Quaternary paleoclimate studies. Earth-Science Reviews 154. 247-278.

Udvardi, B., Kovacs, I.J., Fancsik, T., Konya, P., Batori, M., Stercel, F., Falus, G. and Szalai, Z. 2017. Effects of particle size on the attenuated total reflection spectrum of minerals. Applied Spectroscopy 71. (6): 1157-1168.

VARGA, Gy. and Roettig, C.-B. 2018. Identification of Saharan dust particles in Pleistocene dune sandpaleosol sequences of Fuerteventura (Canary Islands). Hungarian Geographical Bulletin 67. (2): 121-141.

Varga, Gy., Kovács, J., Szalai, Z., Cserháti, C. and ÚJVÁRI, G. 2018. Granulometric characterisation of paleosols in loess series by automated static image analysis. Sedimentary Geology 370. 1-14.

VARGA, Gy., ÚJVÁrI, G. and Kovács, J. 2019a. Interpretation of sedimentary (sub)populations extracted from grain size distributions of Central European loess-paleosol series. Quaternary International 502. 60-70.

Varga, Gy., Gresina, F., Újvári, G., Kovács, J. and SzALAI, Z. 2019b. On the reliability and comparability of laser diffraction grain size measurements of paleosols in loess records. Sedimentary Geology 389. 42-53. 
